\title{
Advances in the three-portal technique for anatomical single- or double-bundle ACL reconstruction
}

\author{
Paulo H. Araujo • Carola F. van Eck • \\ Jeffrey A. Macalena $\cdot$ Freddie H. Fu
}

Received: 23 November 2010/ Accepted: 27 January 2011/Published online: 11 February 2011

(C) The Author(s) 2011. This article is published with open access at Springerlink.com

\begin{abstract}
Purpose To describe the "three-portal technique for anatomical ACL single- or double-bundle reconstruction" and the arthroscopic viewing improvement provided by this technique.

Methods A "high" anterolateral portal was placed $1 \mathrm{~cm}$ lateral to the patellar tendon and the most inferior portion of the portal at the level of the inferior pole of the patella. A "central" portal was placed using a spinal needle under arthroscopic visualization following the orientation of the previous ACL fibers. An accessory medial portal was also placed using a spinal needle respecting a $2-\mathrm{mm}$ distance to the medial femoral condyle.

Results The "high" anterolateral portal permitted a broad and unobstructed view of the ACL tibial attachment. The "central" portal allowed a straightforward view of the ACL femoral remnant and bony landmarks in the intercondylar notch. The accessory medial portal enabled to reach the femoral native insertion site of the ACL.

Conclusion The three-portal technique provides a proper view of the soft tissue remnants and bony landmarks facilitating an anatomical positioning of the graft.
\end{abstract}

Keywords ACL reconstruction - Single bundle $\cdot$ Double bundle $\cdot$ Portal technique

P. H. Araujo - C. F. van Eck · J. A. Macalena · F. H. Fu (凹) Department of Orthopaedic Surgery, University of Pittsburgh, 3471 Fifth Avenue, Kaufman Building Suite 1011, Pittsburgh, PA 15213, USA

e-mail: ffu@upmc.edu

\section{Introduction}

Arthroscopic anterior cruciate ligament (ACL) reconstruction is one of the most often performed orthopedic surgeries [1]. Suboptimum outcomes and arthritic changes in the knee joint after a long-term ACL reconstruction follow-up [2-5] are leading to a change in surgical technique from the traditional transtibial 2-portal technique to the anatomical single- or double-bundle reconstruction 3-portal technique, which more closely restores the native ACL anatomy [6]. Thus, in order to properly place the tibial and femoral tunnels of the ACL, optimal arthroscopic visualization and free triangulation of the instruments are required.

The conventional 2-portal technique uses the anterolateral portal as the viewing portal and the anteromedial portal as the working portal [7]. With this technique, however, a limited view is obtained of the lateral wall of the femoral intercondylar notch, which could result in inaccurate placement of the femoral tunnel(s) (Fig. 1).

Recently, the 3-portal technique is gaining popularity with the increased number of surgeons performing anatomical ACL reconstructions (Fig. 2) [1, 6]. This technique allows interchangeable use of the portals as a viewing and working portals depending on the specific task that is being performed.

The purpose of this study is to describe the correct placement of the portals in the "three-portal technique" and to highlight how the arthroscopic visualization can be improved.

\section{Technical note}

"High" anterolateral portal (LP)

The ACL reconstruction begins with the creation of a "high" anterolateral portal with a number 11 scalpel blade. 
Fig. 1 Arthroscopic view of the right knee in $90^{\circ}$ of flexion.

a Anterolateral portal view (LP) is unable to visualize the femoral ACL insertion site. b Central anteromedial portal view $(\mathrm{CP})$ provides adequate visualization and shows the guide pin is located outside of the native ACL insertion site

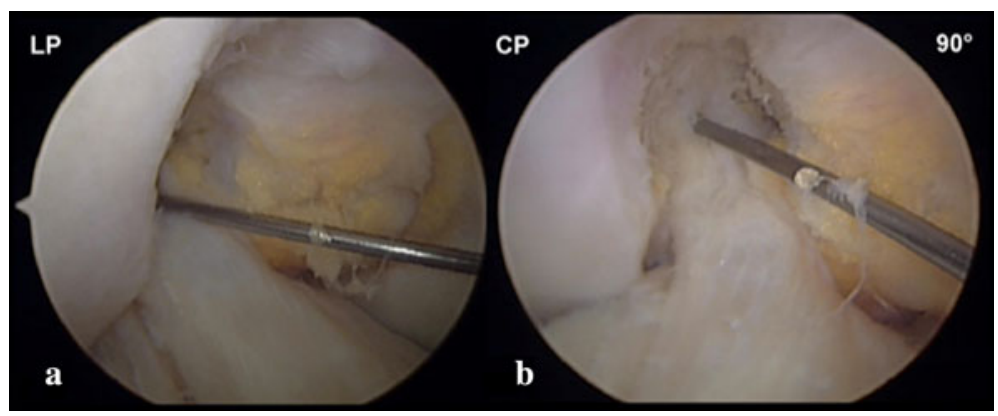

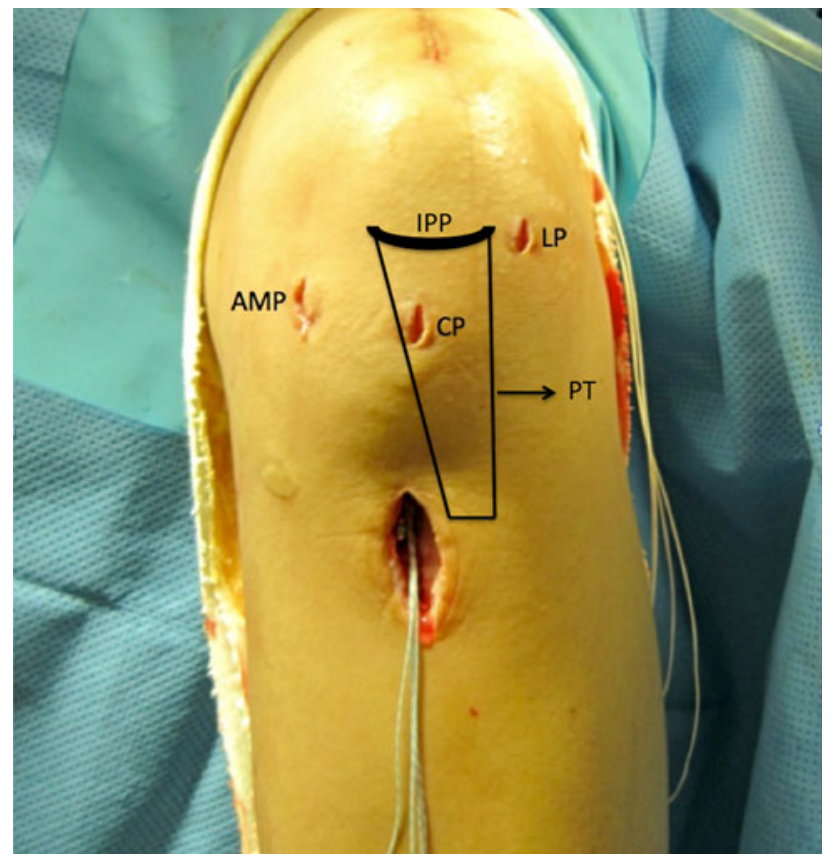

Fig. 2 Left knee in operating position. The three portals and their localization according to the inferior pole of the patella (IPP) and patellar tendon (PT). LP high anterolateral portal, $C P$ central anteromedial portal, $A M P$ accessory anteromedial portal

The blade is oriented with the cutting surface positioned superiorly to avoid damage to the cartilage or meniscus. Its localization is $1 \mathrm{~cm}$ lateral to the patellar tendon, and the most inferior portion of the portal is placed at the level of the inferior pole of the patella when the knee is flexed to $90^{\circ}$. This high position of the portal permits a broad view of the patellofemoral, medial, and lateral compartments and also avoids penetration into Hoffa's fat pad, which is crucial for adequate and unobstructed viewing of the tibial ACL insertion site(s) (Fig. 3).

\section{Central anteromedial portal $(\mathrm{CP})$}

The central anteromedial portal (CP), also called the "central" portal, is the key for an accurate anatomical ACL reconstruction. It allows a straightforward view of the femoral ACL bundles insertion sites that are unlikely visualized with the standard anterolateral portal. Thus, once an adequate view of the lateral intercondylar notch is achieved, there are no indications for additional procedures as notchplasty or wallplasty. This allows preservation of the native bony anatomy and soft tissue remnants, which can be used as landmarks to guide anatomical positioning of the femoral tunnel. (Fig. 4).

Under arthroscopic visualization, a spinal needle is advanced into the joint through the medial third of the patellar tendon just above the joint line. The needle should be in the central portion of the notch in the coronal plane and in the lower $1 / 3$ of the notch with the knee flexed to $90^{\circ}$. A number 11 scalpel blade is advanced vertically through the patellar tendon where the needle perforates the skin. This position allows adequate visualization of the femoral attachment of the ACL (as a viewing portal) and also permits placement of the aiming guide during tibial tunnel drilling (as a working portal). Care should be taken
Fig. 3 a "High" anterolateral portal positioning.

b Arthroscopic view of the tibial ACL insertion site of the right knee from the high anterolateral portal. IPP inferior pole of the patella, $L P$ high anterolateral portal, $A M$ tibial anteromedial bundle insertion site, $P L$ tibial posterolateral bundle insertion site

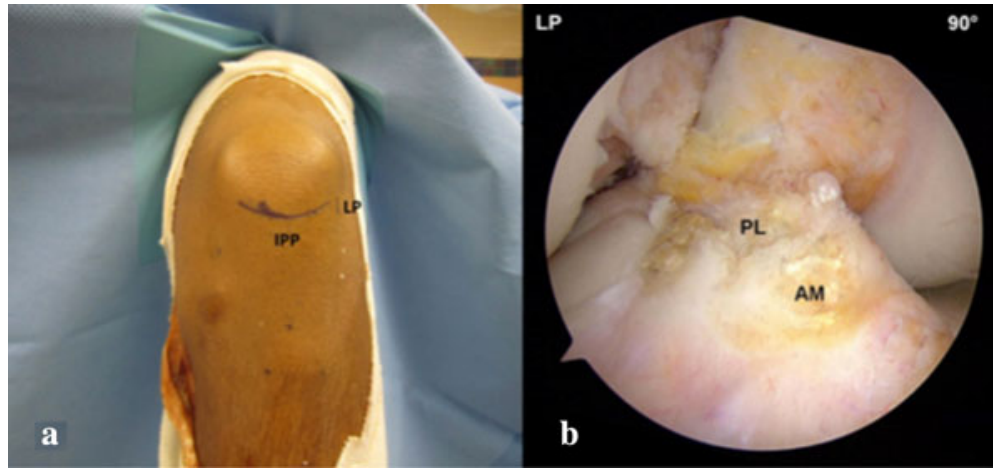




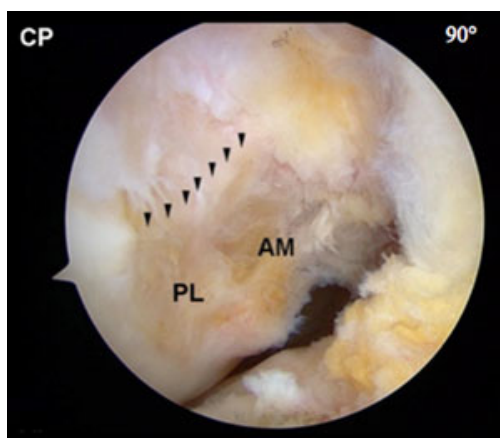

Fig. 4 Central anteromedial portal (CP) view of the femoral insertion site of the right knee. The lateral intercondylar ridge can be visualized, as well as the anteromedial (AM) and posterolateral (PL) bundle insertion sites

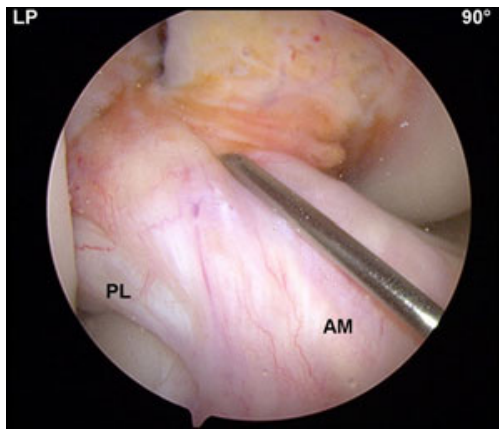

Fig. 5 The central anteromedial portal (CP) is created under direct visualization through the anterolateral (LP) portal and follows the ACL fibers orientation. $A M$ anteromedial, $P L$ posterolateral bundle

not to damage the intermeniscal ligament and articular cartilage during placement of this portal (Fig. 5).

Accessory anteromedial portal (AMP)

The medial portal also called the accessory medial portal (AMP) is mainly used as a working portal for PL and AM femoral tunnel placement. It is noteworthy, however, that the femoral AM tunnel can be drilled, in a transtibial fashion using the PL tibial tunnel around $50 \%$ of the cases and nearly $10 \%$ of the time using the AM tibial tunnel [1].

This portal is created with the knee flexed to $90^{\circ}$. Once again, the use of a spinal needle is essential to the correct placement of the portal. The accessory portal is ordinarily placed above the anterior horn of the medial meniscus approximately $2 \mathrm{~cm}$ medial to the medial border of the patellar tendon. Under arthroscopic visualization through the central portal, a spinal needle is advanced through the proposed position of the medial portal. The spinal needle must pass freely to the anatomical footprint of the ACL in the intercondylar notch. There should be approximately $2 \mathrm{~mm}$ between the spinal needle and the medial femoral condyle to ensure safe drilling (Fig. 6a). Differences in patients' individual anatomy such as notch width will determine the specific location of the AMP.

The AMP is also used as a working portal to place the tibial guide (set at $45^{\circ}$ ) for the tibial PL drilling during double-bundle reconstruction. As a viewing portal, the AMP is used in the measurement of the femoral ACL insertion site with the ruler placed through the LP (Fig. 6 b). After drilling of the femoral tunnel, the AMP can be used, once again, as a viewing portal to evaluate tunnel placement and the integrity of the tunnel and lateral cortex.

\section{Discussion}

The most important findings of the present study are that the internal joint anatomy, as soft tissue remnants and bony landmarks, should guide the placement of the portals in $\mathrm{ACL}$ anatomical reconstruction and that each of the three portals has a specific role during ACL anatomical reconstruction (Table 1).

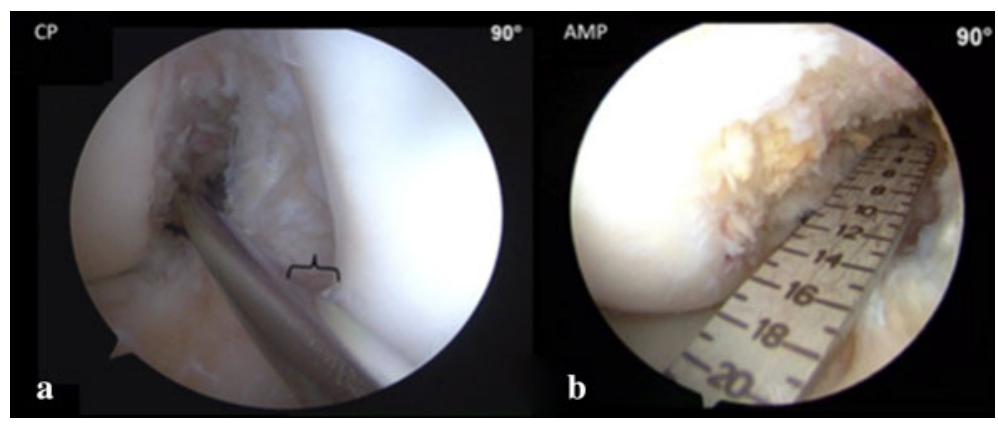

Fig. 6 a Central anteromedial portal $(\mathrm{CP})$ view of the femoral ACL insertion site of the right knee. The guide pin is in the accessory anteromedial portal. There should be at least $2 \mathrm{~mm}$ between the guide pin and the medial femoral condyle (bracket) to ensure safe femoral tunnel drilling without damaging the cartilage. b Under direct visualization through the accessory medial portal, the ruler is inserted through the high anterolateral portal (LP) to measure the femoral ACL insertion site size. $C P$ central anteromedial portal view, $A M P$ accessory medial portal view 
Table 1 Portal usage according to surgical step in anatomical ACL reconstruction

\begin{tabular}{lll}
\hline Surgical step & Viewing portal & Working porta \\
\hline Diagnostic viewing & High anterolateral portal & Central anteromedial portal \\
Tibial ACL footprint measurements & High anterolateral portal & Central anteromedial portal \\
Notch measurements & High anterolateral portal & Central anteromedial portal \\
Femoral ACL footprint measurements & Accessory anteromedial portal & High anterolateral portal \\
PL femoral tunnel placement & Central anteromedial portal & Accessory anteromedial portal \\
PL tibial tunnel placement & High anterolateral portal & Accessory anteromedial portal \\
AM tibial tunnel placement & High anterolateral portal & Central anteromedial portal \\
AM femoral tunnel placement & Central anteromedial portal & Accessory anteromedial portal or transtibial \\
Femoral SB tunnel placement & Central anteromedial portal & Accessory anteromedial portal \\
Tibial SB tunnel placement & High anterolateral portal & Central anteromedial portal \\
\hline
\end{tabular}

$A C L$ Anterior cruciate ligament, $P L$ posterolateral bundle, $A M$ anteromedial bundle, $S B$ single bundle

Failure of ACL reconstruction commonly results from any combination of 3 factors: biologic factors, new traumatic event, and errors of surgical technique [4]. The last one represents the majority of failed ACL reconstructions $[2,8]$.

Among the technical errors, the most common is the tunnel malplacement. This is derived from multiple causes, and poor or limited visualization of the ACL remnants, and bony landmarks certainly plays a prominent role [9].

Lubovitz has described a number of advantages when using the AM portal, instead of the transtibial technique, to drill the femoral tunnels despite the required learning curve [10], which was discussed and reinforced by Snow et al. [5]. Bedi et al. has emphasized that the accurate anatomical placement of the tunnels should be based on footprints and landmarks [2]. All these statements are in accordance with the technique presented in this study.

Restoration of normal anatomy after ACL reconstruction is related to achieve better function and a satisfactory long-term outcomes. The principles for anatomical ACL reconstruction are to functionally reestablish the ACL to its native dimensions, collagen orientation and insertion sites [6]. The basis for the completion of these principles lies in the correct identification of the insertion sites. This study presents an easy method, for surgical daily using, to accomplish this goal.

\section{Conclusion}

The conventional arthroscopic reconstruction of the ACL using a 2-portal technique limits the proper visualization of the tibial and femoral insertion sites. On the other hand, the three-portals technique provides a proper view of the soft tissue remnants and bony landmarks facilitating an anatomical placement of the graft.
Open Access This article is distributed under the terms of the Creative Commons Attribution Noncommercial License which permits any noncommercial use, distribution, and reproduction in any medium, provided the original author(s) and source are credited.

\section{References}

1. Schreiber VM, van Eck CF, Fu FH (2010) Anatomic doublebundle ACL reconstruction. Sports Med Arthrosc Rev 18:27-32

2. Bedi A, Altchek DW (2009) The "Footprint" anterior cruciate ligament technique: an anatomic approach to anterior cruciate ligament reconstruction. Arthroscopy 25:1128-1138

3. Lohmander LS, Ostenberg A, Englund M, Roos H (2004) High prevalence of knee osteoarthritis, pain, and functional limitations in female soccer players twelve years after anterior cruciate ligament injury. Arthritis Rheum 50:3145-3152

4. Nikolaou VS, Efstathopoulos N, Sourlas I, Pilichou A, Papachristou G (2009) Anatomic double-bundle versus single-bundle ACL reconstruction: a comparative biomechanical study in rabbits. Knee Surg Sports Traumatol Arthrosc 17:895-906

5. Snow M, Stanish WD (2010) Double-bundle ACL reconstruction: how big is the learning curve? Knee Surg Sports Traumatol Arthrosc 18:1195-1200

6. van Eck CF, Schreiber VM, Liu TT, Fu FH (2010) The anatomic approach to primary, revision and augumentation anterior cruciate ligament rconstruction. Knee Surg Sports Traumatol Arthrosc 18:1154-1163

7. Cohen SB, Fu FH (2007) Three-portal technique for anterior cruciate ligament reconstruction: use of a central medial portal. Arthroscopy 23:325.e1-325.e4

8. Johnson DL, Swenson TM, Irrgang JJ, Fu FH, Harner CD (1996) Revision anterior cruciate ligament surgery: experience from Pittsburgh. Clin Orthop Relat Res 325:100-109

9. Sommer C, Friederich NF, Müller W (2000) Improperly placesanterior cruciate ligament grafts: correlation between radiological parameters and clinical results. Knee Surg Sports Traumatol Arthrosc 8:207-213

10. Lubowitz JH (2009) Anteromedial portal technique for the anterior cruciate ligament femoral socket: pitfalls and solutions. Arthroscopy 25:95-101 